\title{
Selection of reference genes for transcript profiling of Sargassum polycystum by quantitative real-time polymerase chain reaction
}

\begin{abstract}
Sargassum species are one of the major alginate-producing seaweed species in Asian countries. Alginate is widely used in food, feed, pharmaceutical and medical industries as thickening and stabilizing agents. To establish a set of consistently expressed genes as reference genes for quantitative real-time polymerase chain reaction (qRT-PCR) studies of Sargassum polycystum (Fucales, Ochrophyta) in samples collected at two distinct time points from the field, four candidate reference genes, namely ribosomal protein L3 (RPL3), ribosomal protein S15 (RPS15), alphatubulin ( $\alpha$-TUB) and eukaryotic translation elongation factor 1 alpha (TEF1 $\alpha$ ), were analyzed using geNorm and NormFinder. The results showed that RPL3, $\alpha$-TUB and TEF1 $\alpha$ were the most stable genes using both programs, whereas RPS15 gene was shown to be the least stable. Identification of stably expressed reference genes is crucial for qRT-PCR studies to allow accurate quantification of target gene expression levels. In addition, the expression of key enzyme in the final step of alginate biosynthesis pathway mannuronan C5 epimerase-SP01411 (MC5E-SP01411) and mannuronan C5 epimerase-SP02271 (MC5ESP02271) were differentially expressed in the seaweeds collected at two distinct time points from the field. To our knowledge, this is the first report on validation of reference genes for any Sargassum species. Our data provide a basis for the selection of reference genes for future biological research in related studies.
\end{abstract}

Keyword: Brown algae; Mannuronan C5-epimerase (MC5E); Normalization; qRT-PCR; Reference genes 